qu'il víent d'être dit plus hauł, d'autres poissons d'espèces diverses ont présenté des troubles de même ordre.

Faul-il penser, en présence de ces manifestations indifféremment survenues dans un certain nombre d'espèces et particulières aux sujets ayant été soumis aux courants électriques, à des troubles occasionnés par ces courants dans l'intimité des tissus et notamment dans le labyrinthe audi. tif, troubles capables d'entrainer, au point de vue qui nous intéresse, les plus fâcheuses conséquences ? D'attentives recherches s'imposent à cet égard.

Ainsi apparaissent les éléments du problème sur la direction du poisson par l'emploi du courant électrique. Ces éléments forment un complexe dans lequel interviennent de nombreur facteurs à caractères instables, tels que la température changeante de İ'eau, sa composition variable occasionnant des différences de conductibilité; tels, encore, que les diversités d'espèces de poissons avec leurs sensibililés nerveuses propres, leurs réactions particulières à l'égard de certains facteurs comme les mouvements de l'eau ; les dissemblances de tailles, etc. Vaste ensemble dans lequel surviennent sans cesse des causes incidentes qui diversifient la forme essentielle des phénomènes.

Il semble peut-être possible de concevoir des dispositifs à I'approche desquels chaque poisson vienne évoluer avec sa réaction particulière, sans risque pour sa conservation. Majs, à cause des conditions particulières que présentent nos cours d'eau, du fait qu'ils ne sont généralement petiplés que de petites espèces échappant le plus souvent à l'action des bas voltages, il reste nécessaire de poursuivre des études sur l'appropriation des grilles électriques à nos exigences locales.

\title{
MÉTHODES ET TRUCS DE FÉCONDATION ARTIFICIELLE EN PISCIGULTURE
}

\author{
Par M. Maunice VOUGa \\ Inspecteur gínéral de la pêche, Neuchâtel (Suisse)-
}

(Fin) (1)

Tout ce qui a été dit plus haut se rapporte à des poissons sauvages, capturés vivants dans les eaux libres.

Examinons ce qui arrive lorsque les géniteurs, femelles ou mâles, sont trouvés morts dans les filets tendus sur les places de fraye (lacs ou rivières).

Dans la pratique, il n'est pas toujours possible, à cause de la tempêtc,

(1) Voir Bulletin : - Septembre rg3a, p. $7^{3}$. 
de relever, en temps opportun, des filets tendus en plein lac sur les Irayères des Corégones (féras, palées, bondelles, gravenches, felchen, lavarels, etc.), ou dans les grands profonds, sur les graviers ou les rochers où se passent les amours des Ombles-Chevaliers ou, encore, à l'embouchure des grandes rivières dans les lacs, au moment où l'on cherche à capturer les grandes Truites reproductrices qui, semblables aux Saumons, remontent les cours d'cau en vue de la reproduction. Le poisson emmaillé se débat si longtemps qu'il finit par périr étourté et, lorsque le pisciculteur l'a finalement entre ses mains, ce n'est plus qu'un cadavre dont toute vie a disparu sou. vent depuis plusieurs heures.

Faut-il rejeter ces géniteurs comme impropres à toute pisciculture ?

Certes non.

Toutes les femelles dont les oufs sont bien mûrs, c’est-à-dire hien dîlarliés des grappes ovariennes, peuvent etre frayés post mortem, powr la konme raison que l'eau n'a pas pénétré dans la cavilé abdominale du poisson el que, par conséquent, les culs ne sont pas fon fís. I.e micropyle est encorr. ouvert et si la mort ne date pas de trop longtenips: 7 à xo lieures par exemple, la fécondation de ces $\alpha u f s$ sc fait dans des conditions normales de réussite.

Quant aux mâles, trowés morts aux filets, nous utilisons leur laitance avec succès tant que la mort ne date pas de plus de deux heures. Pour les Truites de lac, nous avons constaté qu'une heure après la mort, la vilalité du sperme diminue considérablement. I a tempéralure de l'air joue éfalement un certain rôle ; par les temps froids, le sperme vit plus longtemps, dans le corps du poisson.

Toutefois, s'il est intéressant de savoir qu'on peut, à l'occasion, féconder des ouls avec la laitance d'un mâle mort, il ne faut faire cetle opération - qu'en cas d'absolue nécessité et se souvenir que la laitance d'un mâle vivant est toujours préférable.

Purisque nous sommes dans un domaine où la pratique joutc un très frand rôle, signalons ici l'importance d'unc totale malurité des oufs et souvenons-nous qu'on peut conserver, sans aucune difficulle, les femelles de certaines espèces de poissons jusqu'à complète maturité sexuclle, en les gardant, le temps voulu, en captivité dans des bassins. Les Truites sont de ce nombre; les Ombles-Chevaliers sont plus difficiles à garder ; tandis que pour les Corégones, les Ombres de rivière, les Brochets, la stabulation en captivité des femelles est très aléatoire. En prenant les précautions voulues, on peut les garder vivantes quelques jours et réussír à les fayer in extremis; mais souvent le poisson souffre trop du changement de milieu et périt sans que ses neuls soient arrivés au degré́ de maturité voulue pour être extraits par la fraye artificielle. Il faut donc s'efforcer, pour ces dernières espèces, de ne pêcher que des poissons mûrs et. pour cela, choisir judicieusement les endroils où tendre les filets.

Souvent les pêcheurs, lorsqu'ils sont directement intéressés à la 
capture des reproducteurs par le produit de la vente des poissons, ne cherchent qu'à prendre Ie plus de sujets possible sans trop s'inquiéter si les femelles seront mûres et, pour cela, tendent leurs filets sur le passage des poissons plutôt que sur les frayères proprement dites. Il en résulte une prise abondante de poissons sans que corresponde une récolte d'cufs proportionnée au nombre des géniteurs. Pourtant un inspecteur connaissant à fond son métier ne se laisse pas prendre deux fois ef sait donner des ordres pour que les filets tendus ramenent une proportion convenable de femelles absolument mûres. Il doit, sans pitié, arrêter tout pêcheur qui ramène une trop forte proportion de fomelles immatures comme par enchantement, l'exemple produit ses fruits et bientôt tout rentre dans lordre.

Dans les lacs suisses, il est tenu une statỉstique complète du nombre des poissons capturés et des cufs obtenus pendant l'époque du frai, pour les especes dont on fait en grand la pisciculture artificielle. On arrive ainsi à établir des moyennes, après quinze, vingt ans de statistigue, et le contrôle du travail piscicole à chaque station se fait aisément par l'écart entre le produit obtenu et la moyenne officielle.

C'est ainsi que, pour la récolte des oufs de Palées au lac de Neuchâtel, on doit obtenir ro.500 oufs en moyenne par femelle pêchéc dans les bords (Palée de bord) et 8.500 à 9.000 cufs par femelle pêchée au Mont (Paléc de fond).

\section{Fraye des Truites arc-en-ciel}

Etudions enfin, dans un dernier chapitre, la manière de travailler les Truites arc-en-ciel conservées comme géniteurs dans les établissements de pisciculture.

Celui qui achète les $œ u f s$ dont il a besoin fait bien, à condition d'avoir un fournisscur consciencicux; mais celui qui produit lui-même les cufs qui Iui sont nécessaires annuellement fait mieux encore, à condition d'opérer selon toutes les règles de l'art.

J'ai vu des centaines de piscicultures, souvent appelé auprès d'elles comme un médecin auprès d'un malade. Eh bien ! à part quelques rares exceplions, $j$ 'ai presque toujours constaté qu'on ne savait pas suffisamment tirer partí des géniteurs arc-en-ciel conservés en captivité, faute de connaftre d'assez près les lois générales de zootechnie.

Ies ouvrages halieutiques ne disent presque rien à ce sujet qui est pourtant d'importance capitale. Les pisciculteurs, livrés à eux-mêmes, éprouvent échecs sur échecs et finissent par abandonner la partie, bien à tort à notre avis.

Comment faut-il faire $p$

Dans toute pisciculture qui se respecte, il faut commencer par sélectionner les élèves, grands et petits.

On choisira, poùr le futiri lot dé reproducteürs, les plus beaux aleving ẹt 
on cherchera à les nourrir d'une part importante de nourriture naturelle (larves, daphnies, asticots, vers de terre, crevettes d'cau douce, ctc.).

Au bout de 6 mois, un premier triage fera mettre de côté les plus forts sujets (mâles et femelles; on peut déjà différencier les sexes à ce momentia), le solde rejoindra le lot de truites de portion.

De 6 a 12 mois, Ies truitclles séicction nées continueront à âtre copieusement nourrics, et, durant toute cette premièrc annéc, il fandra sc souvenir d'un adage bien connu de tous les élcveurs : unc bète qui a souffert dans son' jeune âge est une bêtc irrémédiablement perdue " pour la race".

Au bout d'une année, nouvelle sélection. La dislinction des mâles et des femelles se fait aisément, même pour un profane. On ne conserve alors que le $20 \%$ des mâles emiron, le solde retourne aux truites de portion.

L'annéc suivante ce lot de truites sélectionnécs est soumis à des soins particuliers. Il est alimenté par une prise direcle sur la rivière de manière ì assurer une aération parfaile de l'eau et à permellre l'arrivéc dans l'élanqr d'une bonne proportion de nourribure naturelte; pour bien former te squelelle des futurs jeproducteurs on les nourril le plas possible avec des poissons frais (petits poissons vivants on poissons de mer hachés).

A la fin de la deuvième anméc tous les mâles doivent avoir de la laitance de qualité et être en liabils de noce. On élimine tout ce qui ne serait pas conforme à ce double point te vue airsi que lous les sujess qui ne seraient pas de grosseur suffisanic.

Quant anx femelles, il s'ag̣it de frier soig̣neusement celles qui sont déjà reproductrices de celles momentanement slériles ayant encore la livrée de jeunes poissons. Les premières sont mises à part, il y en a généralement 4 a $5 \%$, quelqueiois plus ; souvent cette proportion n'est pas atteinte.

Ce premier lot de femclles sera l'objet de soins particuliers; elles seront frayées comme il scra dit plus loin, puis replacécs en bassin où unc nourriture copieuse de petits poissons aura tôt fait de les remettre des fatigues de celle première materniti.

Les autres femelles passent l'une après l'autre dans les mains du sélectionneur. Toules celles qui ne sont pas de taille ou de formes irréprochables, toutes celles qui ont des écailles par trop argentées et très facilement détachables vont rejoindje les truites de portion (de vente). En effet, les écailles trop argentées et détachables signifient truites qui dementreront longtemps sterites, $h$ ou 5 ans, même plus. Donc poissons à éliminer, car le pisciculteur doit constamment chercher à avoir des truites qui deviennent reproductrices ; si ce n'est pas à deux ans, comme ce 4 $35 \%$ dont nous venons de parler, tout au moins a 3 ans comme cela doit être la règle pour une honne Arc-en-ciel de race.

Ouvrons ici une parenthèse pour une question financière : une truitc femelle de 3 ans qui pèse 500 grammes donnera environ 1.000 a I.30o nufs; elle vaut comme chair 500 grammes multipliés par le prix de vente, disons 6 fráncs-or du kilo $=3$ francs-or. Mais la valeur de ses œufs est de 7 à 8 francs-or. Donc sa ponte annuelle vaut, pour le piscicul- 
teur, beaucoup plus que la valeur marchande du poisson. Si la femelle a un kilo, la dilférence est encore plus grande : valeur (poids) $=6$ francs-or; valeur de 2.500 oeuls $={ }_{17}, 50$ a 20 lrancs-or. On voit immédiatement quelle importance il y a, pour le pisciculteur, sélectionner les femelles qui donneront des ceufs dès la seconde ou, dans tous les cas, dès la troisième année et à éliminer toutes celles qui ne donneront des ocufs qu'à la quatrième ou à la cinquième année.

Avec un peu d'habitude, on peut donc trier ces femelles de deux ans pour ne garder que les beaux sujets qui arriveront a la maturité sexuelle à la fin de la troisième année. Cela représente, d'habitude, le $70 \%$ du lot, environ. De la deuxième à la troisième année, tout ce lot de géniteurs ou de futurg géniteurs est l'objet d'attentions particulières quant à la nourriture et à l'aération de l'eau. Il est absolument indispensable de donner des poissons d'eau douce ou de mer ; qu'ils soient vivants ou morts, cela n'a pas d'imporlance; mais il est nécessairc, pour la formation des grappes ovariennes, que le phosphate des squelettes des poissons ingérés entre régulièrement dans l'alimentation des truites.

Si le pisciculteur ne peut absolument pas se procurer des petits poissons en quantité suffisante, il devra, alọrs, donner à ses reproducteurs des crerettes d'eau doucc (Gammarus pulex), des larves d'insecies, des vers de terre, des asticots, etc..., qui renferment la chitine et les vitamines indis. pensables à la formation d'une bonne laitance et d'oufs riches en lécitine. Mais nourrir ses reproducteurs comme un lot ordinaire de truites de por* tion, avec des décliets d'abattoirs, des farines fourragères ou de poissons de mer, du sang, du pain ou des macaronis (comme je l'ai vu faire tant de fois) et se figurer que la descendance de ces poissons vaudra quelque chose est une grave erreur.

Nous voici donc en présence, disons au début de Janvier, d'un lot de truites arc-en-cicl de race, spécialement bien nourries. Comment allons: nous faire pour en obtenir les ocufs $p$

Première opénation. - L'étang est vidé, les poissons triés ; les mâles sont mis dans un bassin, les femelles dans un autre. Il faut avoir soin de placer toujours les mâles dans un bassin amont par rapport au bassin des femelles, car il peut arriver, si les sexes ne sont séparés que par un grillage, que les mâles s'excitent et perdent leur laitance s'ils sentent les femelles múres, à quelques centimètres d'eux en amont.

Deuxième opération. - Les femelles sont examinées une à une pour sạvọir și elles peuvent être frayées tout de suite, - dans une dizaine de joụrs, - dạns un mois, - ou encore plus tard ; elles sant triées et placées dạns des bassins différents. Mạis il faut avoir sqin de continuer à les nourrir (de petits poissons vivants, de préférence). C'est une grosse crreur de prétendre, comme beaucoup de pisciculteurs le font encore, qu'il n'est pas nécessaire de nourrir les truites reproductrices à l'approche de la ponte artifícielle. Les cufs et la laitance se ressentent toujours de ce jeûne qui n'est recommandé que trois jours avant la ponte. 
Troisième opération. - Les poissons reconnus bons à frayer sur l'heure sont mis, mảles et femelles, dans une seille de 50 à 60 litres environ, et soumis, pendant une quinzaine de minutes, au bain de formol de LÉGEr (je recommande d'employer une solution plus concentréc que nc l'indique l'jllustre Professeur et, dans nos établissements, nous mettons toujours 6 à 7 centimètres cubes de formol du commerce à $40 \%$, par dix litres d'cau). Puis, tandis que l'aide tient une grossc passoire ou une écuelle percée de petits trous, le praticien saisit une femelle cl lui extrait rapidement tous ses ocufs. Il agit de mème avec une deuxième, puis avec une troisième et une quatrième femclle, par cxemple. Les oculs, toujours recueillis sur lia passoire, sont donc complètement secs. Lorsque la passoirc est pleine, on verse les ceufs dans une curetle ct l'on exprime sur ces cuufs la laitance de 2 ou 3 mâles, on remuc avec une plume d'oic et l'on verse, cufin, le tout dans une cuvette remplie de bonne eat pure et fraiclse ; on remue soignensement et au bout de deux minutes on se met à laver l's cxufs. Cette operation terminée, ils sont mis directement sur les cadres d'incubition.

\section{Pourquoi donner aux gúnitcurs un bain de formol avant la fraye?}

Ce que je vais écrire est le résultat de près tle trente ans d’expéricnces et, malgré l'importance conmerciale du procédé, je n'hésite pas à le publier aujourd'hui et à le donner erratuitement à lous les pisciculteurs, persuadé que je suis de leur rendre un immense service el de leur éviter de nombreux déboires et de grandes pertes de temps.

En conservant des Truites arc-en-ciel en bassins clos, on expose ces poissons à attraper différentes maladies de la peau dont la costiase est la plus fréquente. En frayant ces poissons artificicllement sans bain de formol préventif, on exprime tout le long des flancs des reproducteurs, de Ia femelle surtout, un alondant mucus. Une partic de ce mucus, neuf fois sur dix, tombe sur les acufs ou se mête à la laitance; or, ce mucus renferme le protozoaire flagellé Costia necalrix, arrent direct de la costiase, ou encore d'autres agents d'infection ; les oufs se trouvent ainsi contaminés le jour même de leur récoltc. Malgré tous les soins sulsséquents le pisciculteur voit avec chagrin ses ocuis se piquer d'une pelite tache blanche qui grandil tous les jours et tous les jours il passe quclefues heures de plus à enlever les ocufs gâtés ; bientôt il se décourage et renonce à poursuivre une opération qui ne lui procure que mécomples et chagrins. J'ai vécu ces périodes autrefois et comprends donc tous les sentiments des pisciculteurs à celle phase de leur existence ; mais, actuellement, je puis les assurer que s'ils ont soin de mettre les géniteurs dans le bain de formol avant de les frayer et de désinfecter les mains, les linges et les filets dans le même bain lors de la fraye artificielle, ils éviteront certainement tous nouveaux déboires.

La chose est bien simple; mais il fallait la trouver.

Il reste, enfin, une quatrième opération à faire : opération qui a une 
importance capitale sur la santé des femelles et sur leur avenir de reproductrices. La voici : Quand une femelle est mûre, il faut avoir soin de la frayer jusqu'au bout, bien entendu sans la forcer et en travaillant avec le doigté voulu; puis la remettre rapidement reprendre ses esprits et son souffle dans un bassin d'eau bien courante. La plupart des pisciculteurs ne s'occupent plus de ces femelles jusqu'à l'année suivante. Or qu'arrivet-il P Il reste encore, dans l'abdomen de la femelle, en particulier aux alentours des appendices pyloriques, bon nombre d'ocuís égrenés que la femelle, laissée à elle-même en bassin (même en contact avec des mâles), sera incapable de pondre naturellement. Ces ouls resteront donc dans son corps ; ils prendront, au bout de quelques semaines, une petite lune blanche, puis finiront par être complètement résorbés, momifiés, comme un foctus dans une grossesse extra-utérine; loutefois les cơues demeurent et s'agglomèrent pour former finalement un bouchon qui, à la frayc de l'année suivante, viendra obstruer complètement l'oviducte et entraver considérablement, si ce n'est même empêcher toute nouvelle émission des oufs. Pour éviter ces ennuis, un pisciculteur avisé a soin de reprendre en mains, au bout de 3 ou 4 jours, toutes les femelles travaillées pour les faire frayer une seconde, parfois mème une lroisième fois. L'irritation provoquée par la première ponte artificiclle a pour effet de fairc transsuder, dans la cavité abdominale, un abondant sérum qui facilitera énormément la fraye artificielle suivante et permettra de débarrasser complètement les femelles de leurs derniers ocufs. Ce nettoyarge est indispensable si l'on veut conserver ses femelles en bonne santé et leur permettre de domer, chaque année, une récolte toujours plus abondante. En agissant de cette manière-là, j'ai obtenu de mes femelles, pendant quatre et même cinq ans, des pontes successives produisant, chaque fois, des oufs de toute première qualité.

Une petite remarque en passant. Si l'on fait la seconde pressée du poisson un ou deux jours après la premièrc, les $\propto u$ is récoltés peuvent encorc ctre fécondés; mais si l'on attend plus de huit jours par exemple, les ceufs qui restent sont baignés dans le sérum; ils se gonflent, le micropyle se ferme ; à l'émission ils sont semblables à une sphère bien remplic et nc peuvent plus alors être récondés.

Il faut avoir soin de ne pas frayer ces iemelles, qui passent pour la seconde fois, dans la même cuvette que celles dont on prend les ocufs pour la première fois, car la quantité de sérum qui s'écoule avec les coufs de seconde émission peut provoquer le gonflement des autres oufs ct empêcher, par la suite, la fécondation.

Toutes cés précautions, tous ces petits " trucs" comme disent les pisciculteurs trouvent donc leur justification scientilique et doivent être retenus attentivement par tout technicien qui veut s'éviter des pertes de temps et d'argent; nous avons pensé faire œuvre ulile en les leur signalant. 